\title{
Analysis of Electron Transport Coefficients in Binary Mixtures of TEOS Gas with Kr, Xe, He and Ne Gases for Using in Plasma Assisted Thin- film Deposition
}

\begin{abstract}
Do Anh Tuan ${ }^{\dagger}$
Abstract - The electron transport coefficients in not only pure atoms and molecules but also in the binary gas mixtures are necessary, especially on understanding quantitatively plasma phenomena and ionized gases. Electron transport coefficients (electron drift velocity, density-normalized longitudinal diffusion coefficient, and density-normalized effective ionization coefficient) in binary mixtures of TEOS gas with buffer gases such as $\mathrm{Kr}, \mathrm{Xe}, \mathrm{He}$, and Ne gases, therefore, was analyzed and calculated by a two-term approximation of the Boltzmann equation in the $\mathrm{E} / \mathrm{N}$ range (ratio of the electric field $\mathrm{E}$ to the neutral number density $\mathrm{N})$ of $0.1-1000 \mathrm{Td}\left(1 \mathrm{Td}=10^{-17} \mathrm{~V} . \mathrm{cm}^{2}\right)$. These binary gas mixtures can be considered to use as the silicon sources in many industrial applications depending on mixture ratio and particular application of gas, especially on plasma assisted thin-film deposition.
\end{abstract}

Keywords: TEOS, thin-film, Electron transport coefficient, Plasma assisted thin-film deposition, Boltzmann equation.

\section{Introduction}

Tetraethoxysilane (TEOS), $\mathrm{Si}\left(\mathrm{OC}_{2} \mathrm{H}_{5}\right)_{4}$, which is a gas with non-toxic and non-explosive characteristics; and is mainly used as the silicon source to deposite $\mathrm{SiO}_{2}$-like films by plasma-enhanced chemical vapor deposition (PECVD) in remote microwave oxygen plasma reactors because the deposited films show good step coverage compared with films deposited from $\mathrm{SiH}_{4}$ [1]. A simple model for the oxygen rich $\mathrm{O}_{2}$ and TEOS gas discharge plasmas was constructed $[2,3]$. The electron transport coefficients in TEOS-O $\mathrm{O}_{2}$ and TEOS-Ar mixture gases were calculated and measured [4]. The binary mixtures of TEOS- $\mathrm{N}_{2}$ have been also used to instead of $\mathrm{SiH}_{4}$-based gas mixtures to reduce the dangers of explosion and toxicity while improving quality of the deposition of $\mathrm{SiO}_{2}$ by PECVD. Recently, the electron transport coefficients in TEOS- $\mathrm{N}_{2}$ mixture gases were also calculated [5]. On the other hand, electron transport coefficients in the mixture gases are necessary data to obtain the plasma models.

For all of above reasons along with the lack of measured electron transport coefficients in the binary mixtures of TEOS gas with buffer gases such as $\mathrm{Kr}, \mathrm{Xe}, \mathrm{He}$ and $\mathrm{Ne}$ gases, in this study, we have calculated and analysed electron drift velocity, density-normalized longitudinal diffusion coefficient, ratio of longitudinal diffusion coefficient to electron mobility, Townsend first ionization coefficient using a two-term approximation of the

$\uparrow$ Corresponding Author: Faculty of Electronics and Electrical Engineering, Hung Yen University of Technology and Education, Khoai Chau, Hung Yen, Vietnam. (tuandoanh@utehy.edu.vn) Received: June 24, 2015; Accepted: October 8, 2015
Boltzmann equation over the wide $\mathrm{E} / \mathrm{N}$ range (ratio of the electric field $\mathrm{E}$ to the number density of neutral atoms or molecules $\mathrm{N}$ ) and the reliable electron collision cross sections of the TEOS, $\mathrm{Kr}, \mathrm{Xe}, \mathrm{He}$ and $\mathrm{Ne}$ gases. These results are the first calculations and the best available so far for the quantitative modeling of plasma discharge for processing procedures using these binary mixture gases.

\section{Analysis}

We used the electron swarm method to determine a set of accurate electron collision cross sections for TEOS, TMS, and $\mathrm{BF}_{3}$ molecules $[4,6,7]$. The two-term approximation of the Boltzmann equation for the energy has been applied to calculate the electron transport coefficients in pure gases and binary mixture gases such as electron drift velocity, density-normalized longitudinal diffusion coefficient, ratio of longitudinal diffusion coefficient to electron mobility, Townsend first ionization coefficient over the wide $\mathrm{E} / \mathrm{N}$ range. The electron transport coefficients calculated from the present set of TEOS gas and binary mixtures of TEOS gas with $\mathrm{Ar}$ and $\mathrm{O}_{2}$ gases are agreement with those in experiments [4]. The sets of electron collision cross sections for $\mathrm{Kr}, \mathrm{Xe}, \mathrm{He}$ and $\mathrm{Ne}$ gases were slightly modified to obtain the accurate sets of electron collision cross sections. The electron transport coefficients calculated by using the present sets of these gases are also compared and good agreement with those in experiments.

In the present study, the electron collision cross sections for TEOS molecule determined by Tuan and Jeon [4]. This 
set includes one momentum transfer, one attachment, two vibrational excitation cross sections (threshold energies of 0.16 and $0.37 \mathrm{eV}$ ), one dissociation excitation (threshold energy of $3.6 \mathrm{eV}$ ), one electronic excitation (threshold energy of $1.7 \mathrm{eV}$ ), and one total ionization (threshold energy of $10.6 \mathrm{eV})$. The set of electron collision cross sections for $\mathrm{Kr}$ atom determined by Hayashi [10] includes one momentum transfer, fourteen electronic excitation cross sections (threshold energies of 9.915 to $13.437 \mathrm{eV}$ ), and one total ionization (threshold energy of $14.0 \mathrm{eV}$ ) cross section. The set of electron collision cross sections for Xe atom determined by Hashimoto and Nakamura [11] includes one momentum transfer, fourteen electronic excitation cross sections (threshold energies of 8.315 to $11.58 \mathrm{eV}$ ), and one total ionization (threshold energy of $12.13 \mathrm{eV}$ ) cross section. The set of electron collision cross sections for $\mathrm{He}$ atom determined by Hayashi [10] have been used as initial sets. This set includes one momentum transfer, twenty five electronic excitation cross sections (threshold energies of 19.82 to $24.19 \mathrm{eV}$ ), and one total ionization (threshold energy of $24.59 \mathrm{eV}$ ) cross section. The set of electron collision cross sections for $\mathrm{Ne}$ atom determined by Hayashi [10] have been used as initial sets. This set includes one momentum transfer, seven electronic excitation cross sections (threshold energies of 16.62 to $19.66 \mathrm{eV}$ ), and one total ionization (threshold energy of $21.56 \mathrm{eV}$ ) cross section. A brief summary of several reaction processes of TEOS molecule and other gases for plasma modeling is listed in Table 1.

The present two-term approximation of the Boltzmann equation for the energy given by Tagashira et al. [12] was also previously used for the pure gases and binary mixture gases [4-9]. The centre-of-mass drift velocity of an electron swarm $\left(\mathrm{W}_{\mathrm{r}}\right)$ and the longitudinal diffusion coefficient $\left(\mathrm{D}_{\mathrm{L}}\right)$ are calculated in the time-of-flight (TOF) parameters. The diffusion-corrected ionization and attachment coefficients are also calculated in the steady state Townsend (SST) parameters. A description of the Boltzmann equation analysis in the previous publications [4-9, 12-16] is also briefly following represented.

The electron transport coefficients in given gases are functions only of the ratio $\mathrm{E} / \mathrm{N}$, the gas temperature $\mathrm{T}$, and when a magnetic field is present, of $\mathrm{B} / \mathrm{N}$. They are related to the electron collision cross sections by complex integral expressions involving the electron energy distribution function (EEDF) [13]. The electron energy distribution function $(\mathrm{EEDF}), \mathrm{f}(\varepsilon, \mathrm{E} / \mathrm{N})$, is normalized by

$$
\int_{0}^{\infty} f(\varepsilon, E / N) d \varepsilon \cong 1
$$

where $\varepsilon$ is the electron energy. Electron transport properties directly depend on EEDF [13]. The EEDF is normally non-Maxwellian in the non-equilibrium plasma used for applications. The EEDF can be obtained theoretically by solving the Boltzmann Eq. [13]

$$
\frac{\partial f}{\partial t}+\vec{v} \nabla_{r} f+\vec{a} \nabla_{v} f=\left(\frac{\partial f}{\partial t}\right)_{\text {coll }}
$$

\begin{tabular}{|c|c|c|c|}
\hline Types of collision & Reactions & Threshold energies $(\mathrm{eV})$ & References and comments \\
\hline \multicolumn{4}{|c|}{$*$ In TEOS molecule } \\
\hline Momentum transfer & $\mathrm{Si}\left(\mathrm{OC}_{2} \mathrm{H}_{5}\right)_{4}+\mathrm{e} \rightarrow \mathrm{Si}\left(\mathrm{OC}_{2} \mathrm{H}_{5}\right)_{4}+\mathrm{e}$ & 0 & Ref. 4 \\
\hline Vibrational excitation & $\mathrm{Si}\left(\mathrm{OC}_{2} \mathrm{H}_{5}\right)_{4}+\mathrm{e} \rightarrow \mathrm{Si}\left(\mathrm{OC}_{2} \mathrm{H}_{5}\right)_{4}(\mathrm{v}=1)+\mathrm{e}$ & 0.16 & Ref. 4 \\
\hline Vibrational excitation & $\mathrm{Si}\left(\mathrm{OC}_{2} \mathrm{H}_{5}\right)_{4}+\mathrm{e} \rightarrow \mathrm{Si}\left(\mathrm{OC}_{2} \mathrm{H}_{5}\right)_{4}(v=2)+\mathrm{e}$ & 0.37 & Ref. 4 \\
\hline Electronic excitation & $\mathrm{Si}\left(\mathrm{OC}_{2} \mathrm{H}_{5}\right)_{4}+\mathrm{e} \rightarrow \mathrm{Si}\left(\mathrm{OC}_{2} \mathrm{H}_{5}\right)_{4}{ }^{*}+\mathrm{e}$ & 1.7 & Ref. 4 \\
\hline Dissociation excitation & $\mathrm{Si}\left(\mathrm{OC}_{2} \mathrm{H}_{5}\right)_{4}+\mathrm{e} \rightarrow \mathrm{Si}\left(\mathrm{OC}_{2} \mathrm{H}_{5}\right)_{4}{ }^{*}+\mathrm{e}$ & 3.6 & Refs. 4 and 17 \\
\hline Ionization & $\mathrm{Si}\left(\mathrm{OC}_{2} \mathrm{H}_{5}\right)_{4}+\mathrm{e} \rightarrow \mathrm{Si}\left(\mathrm{OC}_{2} \mathrm{H}_{5}\right)_{4}^{+}+\mathrm{e}$ & 10.6 & Refs. 4,17 , and 18 \\
\hline \multicolumn{4}{|c|}{$*$ In $\mathrm{Kr}$ atom } \\
\hline Momentum transfer & $\mathrm{Kr}+\mathrm{e} \rightarrow \mathrm{Kr}+\mathrm{e}$ & 0 & Ref. 10 \\
\hline Electronic excitation & $\mathrm{Kr}+\mathrm{e} \rightarrow \mathrm{Kr}^{*}+\mathrm{e}$ & $9.915 \div 13.437$ & $\begin{array}{l}\text { Ref. 10, includes fourteen } \\
\text { electronic excitations }\end{array}$ \\
\hline Ionization & $\mathrm{Kr}+\mathrm{e} \rightarrow \mathrm{Kr}^{+}+\mathrm{e}$ & 14.0 & Ref. 10 \\
\hline \multicolumn{4}{|c|}{$*$ In Xe atom } \\
\hline Momentum transfer & $\mathrm{Xe}+\mathrm{e} \rightarrow \mathrm{Xe}+\mathrm{e}$ & 0 & Ref. 11 \\
\hline Electronic excitation & $\mathrm{Xe}+\mathrm{e} \rightarrow \mathrm{Xe}^{*}+\mathrm{e}$ & $8.315 \div 11.58$ & $\begin{array}{l}\text { Ref. 11, includes fourteen } \\
\text { electronic excitations }\end{array}$ \\
\hline Ionization & $\mathrm{Xe}+\mathrm{e} \rightarrow \mathrm{Xe}^{+}+\mathrm{e}$ & 12.13 & Ref. 11 \\
\hline \multicolumn{4}{|c|}{$*$ In He atom } \\
\hline Momentum transfer & $\mathrm{He}+\mathrm{e} \rightarrow \mathrm{He}+\mathrm{e}$ & 0 & Ref. 10 \\
\hline Electronic excitation & $\mathrm{He}+\mathrm{e} \rightarrow \mathrm{He}^{*}+\mathrm{e}$ & $19.82 \div 24.19$ & $\begin{array}{l}\text { Ref. } 10 \text {, includes twenty five } \\
\text { electronic excitations }\end{array}$ \\
\hline Ionization & $\mathrm{He}+\mathrm{e} \rightarrow \mathrm{He}^{+}+\mathrm{e}$ & 24.59 & Ref. 10 \\
\hline \multicolumn{4}{|c|}{$*$ In Ne atom } \\
\hline Momentum transfer & $\mathrm{Ne}+\mathrm{e} \rightarrow \mathrm{Ne}+\mathrm{e}$ & 0 & Ref. 10 \\
\hline Electronic excitation & $\mathrm{Ne}+\mathrm{e} \rightarrow \mathrm{Ne}^{*}+\mathrm{e}$ & $16.62 \div 19.66$ & $\begin{array}{l}\text { Ref. 10, includes seven } \\
\text { electronic excitations }\end{array}$ \\
\hline Ionization & $\mathrm{Ne}+\mathrm{e} \rightarrow \mathrm{Ne}^{+}+\mathrm{e}$ & 21.56 & Ref. 10 \\
\hline
\end{tabular}

Table 1. Reaction processes with threshold energy for plasma modeling in atoms and molecules 
where $\mathrm{f}=\mathrm{f}(\vec{r}, \vec{v}, t)$ is the distribution function of the positions $\mathrm{r}$ and velocities of electrons $\mathrm{v}, \vec{a}$ is the acceleration due to external forces, and $(\partial \mathrm{f} / \partial \mathrm{t})_{\text {coll }}$ is the collision induced rate of change of the number of electrons per unit volume of phase space. The solution normally involves the assumptions that the fields are time independent, that spatial gradient terms can be omitted, and that the distribution in velocity space is only slightly disturbed from spherical.

The electron drift velocity calculated from the solution of electron energy distribution function, $\mathrm{f}(\varepsilon, \mathrm{E} / \mathrm{N})$, of the Boltzmann equation is defined as

$$
W=-\frac{1}{3}\left(\frac{2}{m}\right)^{1 / 2} \frac{e E}{N} \int_{0}^{\infty} \frac{\varepsilon}{q_{m}(\varepsilon)} \frac{d f(\varepsilon, E / N)}{d \varepsilon} d \varepsilon
$$

where $\mathrm{m}$ is the electron mass, $\mathrm{e}$ is the elementary charge and $\mathrm{q}_{\mathrm{m}}(\varepsilon)$ is the momentum-transfer cross section.

The density-normalized longitudinal diffusion coefficient is defined as

$$
\begin{array}{r}
N D_{L}=\frac{V_{1}}{3 N}\left(E \int_{0}^{\infty} \frac{\varepsilon}{q_{T}} \frac{\partial}{\partial \varepsilon}\left(F_{1} \varepsilon^{-1 / 2}\right) d \varepsilon+\int_{0}^{\infty} \frac{\varepsilon^{1 / 2}}{q_{T}} F_{0} d \varepsilon\right) \\
-\left(\varpi_{0} A_{2}-\varpi_{1} A_{1}-\varpi_{02}\right) .
\end{array}
$$

where $\mathrm{V}_{1}$ is the speed of electron, $\mathrm{q}_{\mathrm{T}}$ is the total cross section, here $\mathrm{F}_{\mathrm{n}}$ and $\varpi_{n}(\mathrm{n}=0,1,2)$ are respectively the electron energy distributions of various orders and their eigen values. $\mathrm{V}_{1}, \varpi_{n}, \varpi_{0 n}$, and $\mathrm{A}_{\mathrm{n}}$ are given by

$$
\begin{gathered}
V_{1}=\left(\frac{2 e}{m}\right)^{1 / 2} \\
\varpi_{0}=V_{1} N \int_{0}^{\infty} \varepsilon^{1 / 2} q_{i} F_{0} d \varepsilon
\end{gathered}
$$

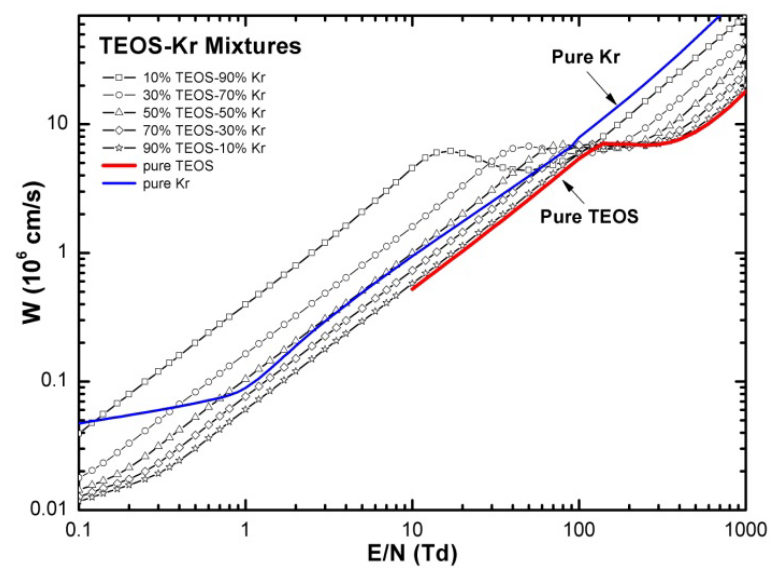

Fig. 1. Electron drift velocity, $\mathrm{W}$, as functions of $\mathrm{E} / \mathrm{N}$ for the TEOS-Kr mixtures with $10 \%, 30 \%, 50 \%, 70 \%$, and $90 \%$ TEOS. The solid curves show present $\mathrm{W}$ values calculated for the pure $\mathrm{Kr}$ atom and TEOS molecule.

$$
\begin{gathered}
\varpi_{1}=-\frac{V_{1} E}{3 N} \int_{0}^{\infty} \frac{\varepsilon}{q_{T}} \frac{\partial}{\partial \varepsilon}\left(F_{0} \varepsilon^{-1 / 2}\right) d \varepsilon+\left(\varpi_{0} A_{1}-\varpi_{01}\right) \\
\varpi_{0 n}=V_{1} N \int_{0}^{\infty} \varepsilon^{1 / 2} q_{i} F_{n} d \varepsilon \\
A_{n}=\int_{0}^{\infty} F_{n} d \varepsilon
\end{gathered}
$$

where $\mathrm{q}_{\mathrm{i}}$ is the ionization cross section.

The Townsend first ionization coefficient is defined as

$$
\alpha / N=\frac{1}{W}\left(\frac{2}{m}\right)^{1 / 2} \int_{I}^{\infty} f(\varepsilon, E / N) \varepsilon^{1 / 2} q_{i}(\varepsilon) d \varepsilon
$$

where $I$ is the ionization onset energy and $q_{i}(\varepsilon)$ is the ionization cross section.

\section{Electron transport coefficients}

The electron drift velocities, $\mathrm{W}$, the density-normalized longitudinal diffusion coefficients, $\mathrm{ND}_{\mathrm{L}}$, the ratio of the longitudinal diffusion coefficients to the electron mobility, $\mathrm{D}_{\mathrm{L}} / \mu$, the Townsend first ionization coefficients, $\alpha / \mathrm{N}$, as functions of $\mathrm{E} / \mathrm{N}$ for the binary mixtures of TEOS gas with other gases such as $\mathrm{Kr}, \mathrm{Xe}, \mathrm{He}$ and $\mathrm{Ne}$ have been calculated in the $\mathrm{E} / \mathrm{N}$ range $0.1<\mathrm{E} / \mathrm{N}<1000 \mathrm{Td}$ by a twoterm approximation of the Boltzmann equation for energies.

The calculated electron drift velocities in TEOS-Kr, TEOS-Xe, TEOS-He, and TEOS-Ne mixture gases are shown in Figs. 1-4. In Figs. 1 and 2, the $\mathrm{W}$ values in TEOS-Kr and TEOS-Xe mixture gases are suggested to be between those of the pure gases over the range of $\mathrm{E} / \mathrm{N}>$ $100 \mathrm{Td}$. In the range of $\mathrm{E} / \mathrm{N}<100 \mathrm{Td}$, the $\mathrm{W}$ values in these binary mixtures are higher than those of $\mathrm{Kr}, \mathrm{Xe}$, and TEOS gases, especially on $\mathrm{E} / \mathrm{N}=10 \mathrm{Td}$ corresponding to electron energy of about $0.202 \mathrm{eV}$. To the best of our

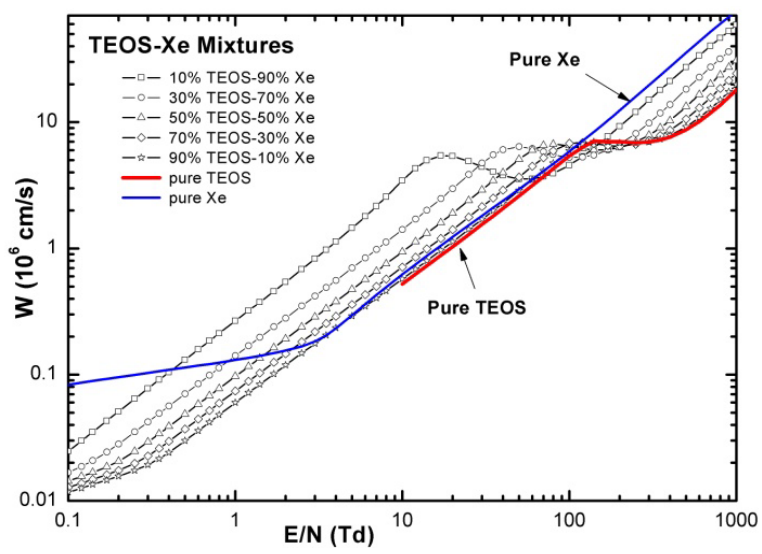

Fig. 2. Electron drift velocity, $\mathrm{W}$, as functions of $\mathrm{E} / \mathrm{N}$ for the TEOS-Xe mixtures with $10 \%, 30 \%, 50 \%, 70 \%$, and $90 \%$ TEOS. The solid curves show present $\mathrm{W}$ values calculated for the pure Xe atom and TEOS molecule. 


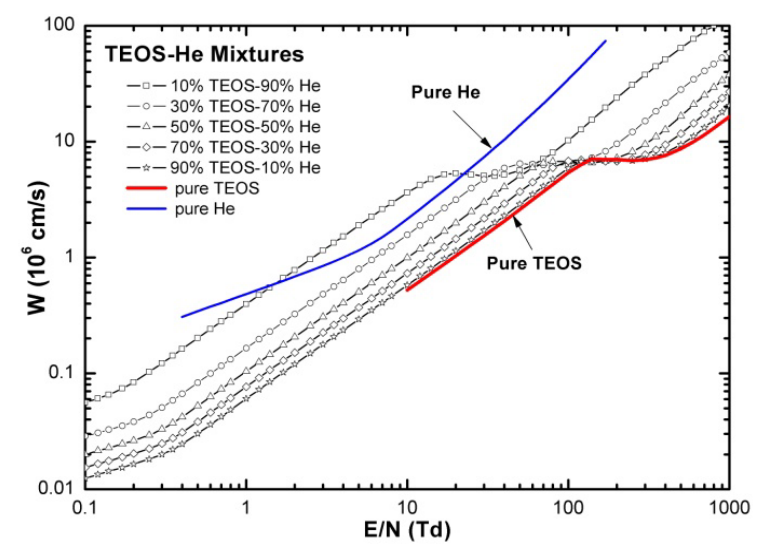

Fig. 3. Electron drift velocity, $\mathrm{W}$, as functions of $\mathrm{E} / \mathrm{N}$ for the TEOS-He mixtures with $10 \%, 30 \%, 50 \%, 70 \%$, and $90 \%$ TEOS. The solid curves show present $\mathrm{W}$ values calculated for the pure $\mathrm{He}$ atom and TEOS molecule.

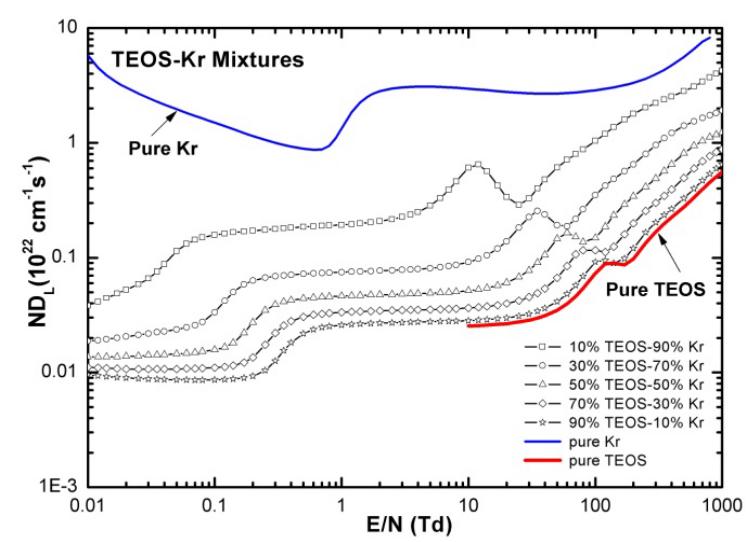

Fig. 5. Density-normalized longitudinal diffusion coefficient, $\mathrm{ND}_{\mathrm{L}}$, as functions of $\mathrm{E} / \mathrm{N}$ for the TEOS-Kr mixtures with $10 \%, 30 \%, 50 \%, 70 \%$, and $90 \%$ TEOS. The solid curves show present $\mathrm{ND}_{\mathrm{L}}$ values calculated for the pure TEOS molecule and $\mathrm{Kr}$ atom.

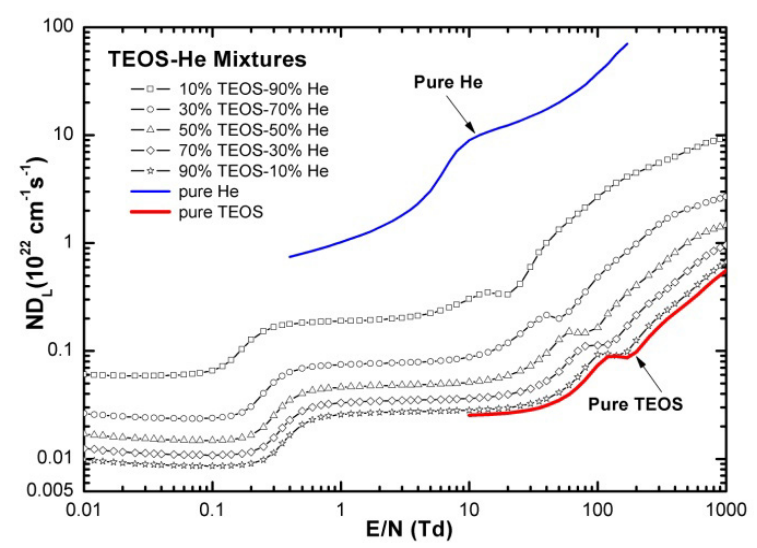

Fig. 7. Density-normalized longitudinal diffusion coefficient, $\mathrm{ND}_{\mathrm{L}}$, as functions of $\mathrm{E} / \mathrm{N}$ for the TEOS-He mixtures with $10 \%, 30 \%, 50 \%, 70 \%$, and $90 \%$ TEOS. The solid curves show present $\mathrm{ND}_{\mathrm{L}}$ values calculated for the pure TEOS molecule and He atom.

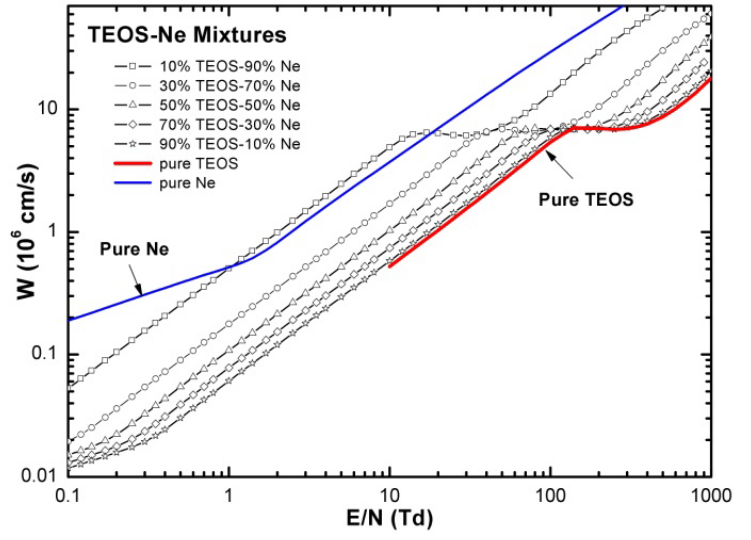

Fig. 4. Electron drift velocity, $\mathrm{W}$, as functions of $\mathrm{E} / \mathrm{N}$ for the TEOS-Ne mixtures with $10 \%, 30 \%, 50 \%, 70 \%$, and $90 \%$ TEOS. The solid curves show present $\mathrm{W}$ values calculated for the pure $\mathrm{Ne}$ atom and TEOS molecule.

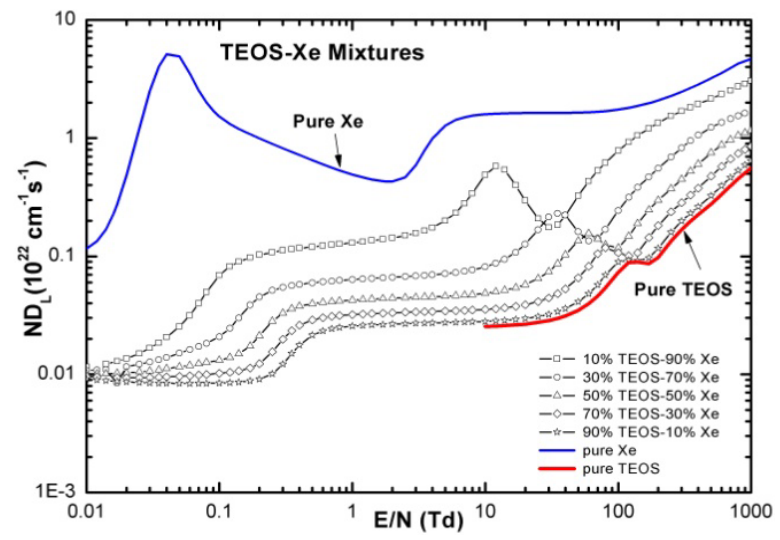

Fig. 6. Density-normalized longitudinal diffusion coefficient, $\mathrm{ND}_{\mathrm{L}}$, as functions of $\mathrm{E} / \mathrm{N}$ for the TEOS-Xe mixtures with $10 \%, 30 \%, 50 \%, 70 \%$, and $90 \%$ TEOS. The solid curves show present $\mathrm{ND}_{\mathrm{L}}$ values calculated for the pure TEOS molecule and Xe atom.

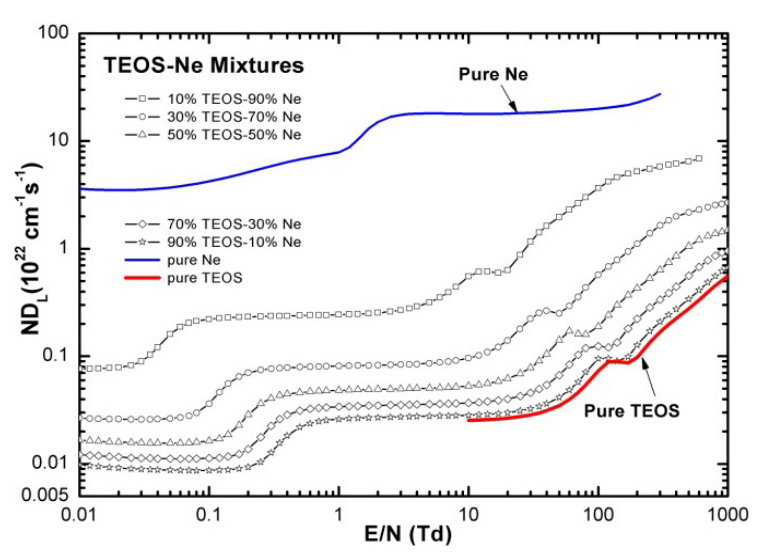

Fig. 8. Density-normalized longitudinal diffusion coefficient, $\mathrm{ND}_{\mathrm{L}}$, as functions of $\mathrm{E} / \mathrm{N}$ for the TEOS-Ne mixtures with $10 \%, 30 \%, 50 \%, 70 \%$, and $90 \%$ TEOS. The solid curves show present $\mathrm{ND}_{\mathrm{L}}$ values calculated for the pure TEOS molecule and $\mathrm{Ne}$ atom. 
knowledge, the electron drift velocity strongly depends onmomentum transfer cross sections and vibrational excitation cross sections. In these cases, the reasons could be suggested that at this energy the vibrational excitations in TEOS gas occured, the momentum transfer cross section of TEOS gas is higher than that of $\mathrm{Kr}$ and $\mathrm{Xe}$ gases and the momentum transfer cross sections of $\mathrm{Kr}$ and $\mathrm{Xe}$ gases are deeply decreasing to the minimum points. Therefore, these curves have the same tendency as that of pure TEOS gas. For TEOS-He and TEOS-Ne mixture gases in Figs. 2 and 4, the $\mathrm{W}$ values are suggested to be between those of the pure gases over the all range of $\mathrm{E} / \mathrm{N}$. However, the $\mathrm{W}$ values of $10 \%$ TEOS-He and 10\% TEOS-Ne mixtures are higher than those of pure TEOS, $\mathrm{He}$, and $\mathrm{Ne}$ gases in the range of $\mathrm{E} / \mathrm{N}<20 \mathrm{Td}$ (corresponding to electron energy of about

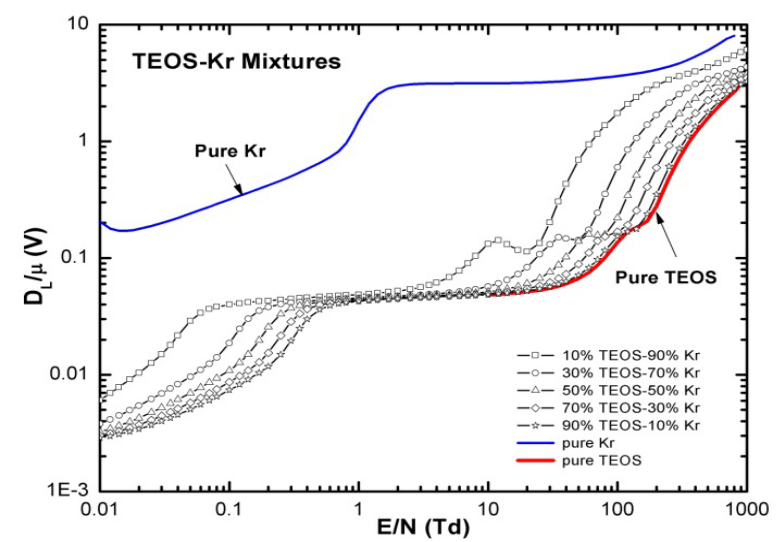

Fig. 9. Ratios of longitudinal diffusion coefficient to the electron mobility, $\mathrm{D}_{\mathrm{L}} / \mu$, as functions of $\mathrm{E} / \mathrm{N}$ for the TEOS-Kr mixtures with $10 \%, 30 \%, 50 \%, 70 \%$, and $90 \%$ TEOS. The solid curves show present $\mathrm{D}_{\mathrm{L}} / \mu$ values calculated for the pure TEOS molecule and $\mathrm{Kr}$ atom.

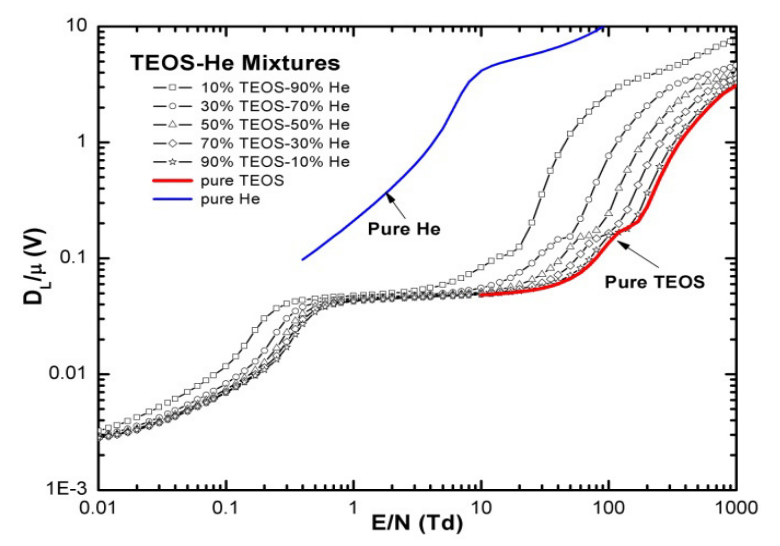

Fig. 11. Ratios of longitudinal diffusion coefficient to the electron mobility, $D_{L} / \mu$, as functions of $E / N$ for the TEOS-He mixtures with $10 \%, 30 \%, 50 \%, 70 \%$, and $90 \%$ TEOS The solid curves show present $\mathrm{D}_{\mathrm{L}} / \mu$ values calculated for the pure TEOS molecule and He atom.
$0.202 \mathrm{eV}$ of about $0.15 \mathrm{eV})$. In these cases, the reasons could be suggested that at this energy the momentum transfer cross section of TEOS gas is higher than that of He and $\mathrm{Ne}$ gases and the momentum transfer cross sections of $\mathrm{He}$ and Ne gases are slightly increasing.

The calculated density-normalized longitudinal diffusion coefficients and calculated ratio of the longitudinal diffusion coefficients to the electron mobility in TEOS-Kr, TEOS-Xe, TEOS-He, and TEOS-Ne mixture gases are shown in Figs. 5-8 and Figs. 9-12, respectively. The calculated values of these coefficients in the binary mixtures of TEOS with $\mathrm{Kr}$, $\mathrm{Xe}, \mathrm{He}$, and $\mathrm{Ne}$ gases are suggested to be between those of the pure gases over the all range of $\mathrm{E} / \mathrm{N}$.

The calculated Townsend first ionization coefficients in TEOS-Kr, TEOS-Xe, TEOS-He, and TEOS-Ne mixture

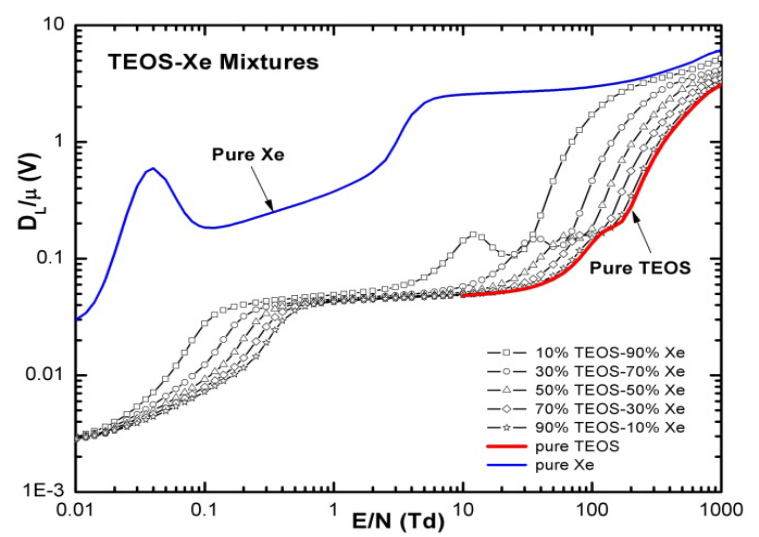

Fig. 10. Ratios of longitudinal diffusion coefficient to the electron mobility, $\mathrm{D}_{\mathrm{L}} / \mu$, as functions of $\mathrm{E} / \mathrm{N}$ for the TEOS-Xe mixtures with $10 \%, 30 \%, 50 \%, 70 \%$, and $90 \%$ TEOS. The solid curves show present $\mathrm{D}_{\mathrm{L}} / \mu$ values calculated for the pure TEOS molecule and Xe atom.

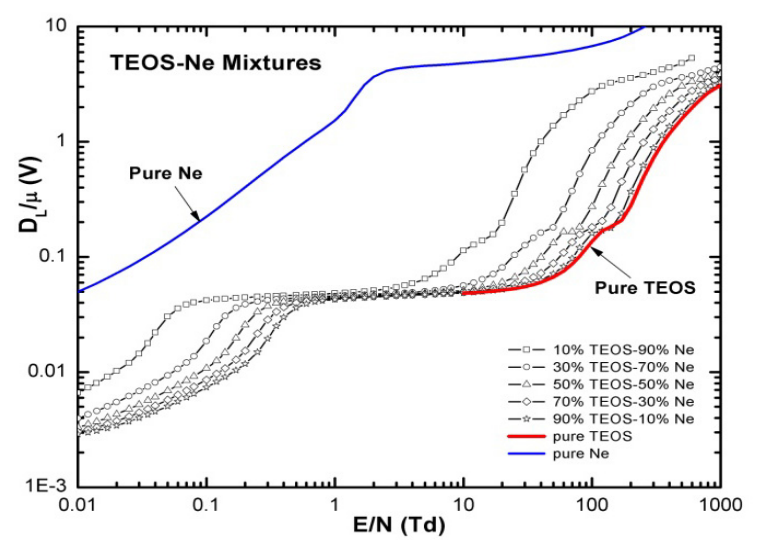

Fig. 12. Ratios of longitudinal diffusion coefficient to the electron mobility, $\mathrm{D}_{\mathrm{L}} / \mu$, as functions of $\mathrm{E} / \mathrm{N}$ for the TEOS-Ne mixtures with $10 \%, 30 \%, 50 \%, 70 \%$, and $90 \%$ TEOS The solid curves show present $\mathrm{D}_{\mathrm{L}} / \mu$ values calculated for the pure TEOS molecule and $\mathrm{Ne}$ atom. 
gases are shown in Figs. 13-16. In Figs. 13 and 14, the $\alpha / \mathrm{N}$ values in TEOS- $\mathrm{Kr}$ and TEOS-Xe mixture gases are suggested to be between those of the pure gases over the ranges of $\mathrm{E} / \mathrm{N}<200 \mathrm{Td}$ and $\mathrm{E} / \mathrm{N}<700 \mathrm{Td}$, respectively. The $\alpha / \mathrm{N}$ values in $10 \%$ TEOS-Kr and $10 \%$ TEOS-Xe gas mixtures are closed to those of pure $\mathrm{Kr}$ and $\mathrm{Xe}$ gases. These values could be greater than those of pure $\mathrm{Kr}$ and $\mathrm{Xe}$ gases over the ranges of $\mathrm{E} / \mathrm{N}>200 \mathrm{Td}$ and $\mathrm{E} / \mathrm{N}>700 \mathrm{Td}$, respectively.

To the best of our knowledge, the Townsend first ionization coefficient strongly depends on ionization cross sections and dissociation excitation cross sections. The reasons could be suggested that the ionization and dissociation excitation cross sections of TEOS gas are much higher than those of $\mathrm{Kr}$ and Xe gases. For TEOS-He and TEOS-Ne mixture gases in Figs. 15 and 16, the $\alpha / \mathrm{N}$ values are suggested to be between those of the pure gases

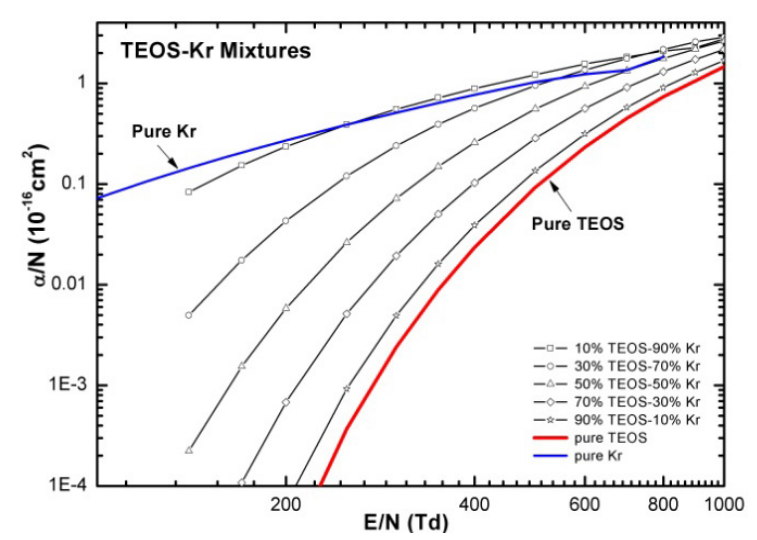

Fig. 13. The Townsend first ionization coefficients, $\alpha / \mathrm{N}$, as functions of $\mathrm{E} / \mathrm{N}$ for the TEOS-Kr mixtures with $10 \%, 30 \%, 50 \%, 70 \%$, and $90 \%$ TEOS. The solid curves show present $\alpha / \mathrm{N}$ values calculated for the pure TEOS and $\mathrm{Kr}$ molecules.

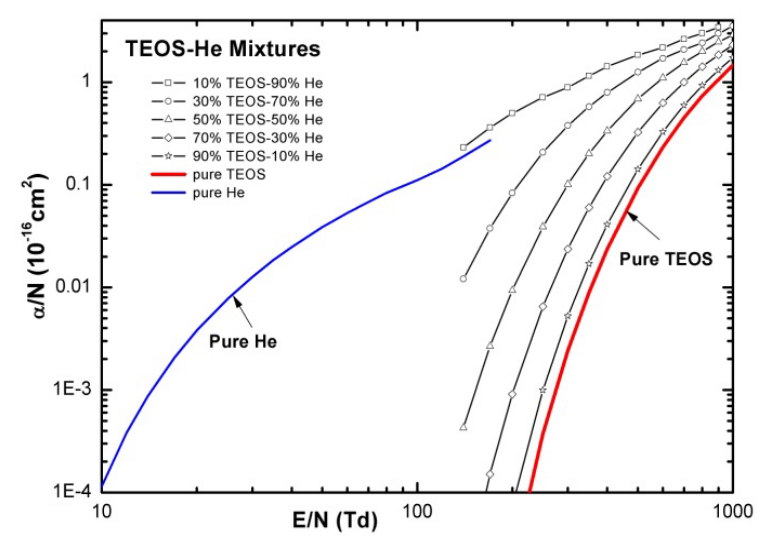

Fig. 15. The Townsend first ionization coefficients, $\alpha / \mathrm{N}$, as functions of $\mathrm{E} / \mathrm{N}$ for the TEOS-He mixtures with $10 \%, 30 \%, 50 \%, 70 \%$, and $90 \%$ TEOS The solid curves show present $\alpha / \mathrm{N}$ values calculated for the pure TEOS and He molecules. over the all range of $\mathrm{E} / \mathrm{N}$. However, the $\alpha / \mathrm{N}$ values of $10 \%$ TEOS-He and 10\% TEOS-Ne mixtures are higher than those of pure TEOS, $\mathrm{He}$, and $\mathrm{Ne}$ gases over the all range of $\mathrm{E} / \mathrm{N}$. In these cases, the reasons could be suggested that ionization cross sections of $\mathrm{He}$ and $\mathrm{Ne}$ gases are much lower than that of TEOS gas.

The present results, that have been also calculated for the first time, are reliable over the $\mathrm{E} / \mathrm{N}$ range of 0.1 to $1000 \mathrm{Td}$ because of the accuracy of the electron collision cross sections for the present gases and the validity of the Boltzmann equation. More experiments of the electron transport coefficients for these binary mixtures need to be performed over the wide range of $\mathrm{E} / \mathrm{N}$ in the future. From ionization coefficients of these binary mixtures, we could make the source of plasma corresponding equivalent industrial applications in manufacturing nano-material, depending on mixture ratio and particular application of

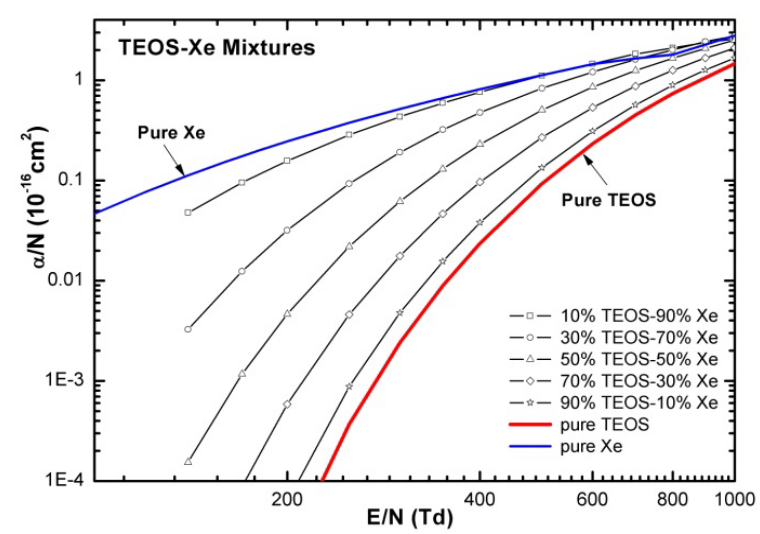

Fig. 14. The Townsend first ionization coefficients, $\alpha / \mathrm{N}$, as functions of $\mathrm{E} / \mathrm{N}$ for the TEOS-Xe mixtures with $10 \%, 30 \%, 50 \%, 70 \%$, and $90 \%$ TEOS. The solid curves show present $\alpha / \mathrm{N}$ values calculated for the pure TEOS and Xe molecules.

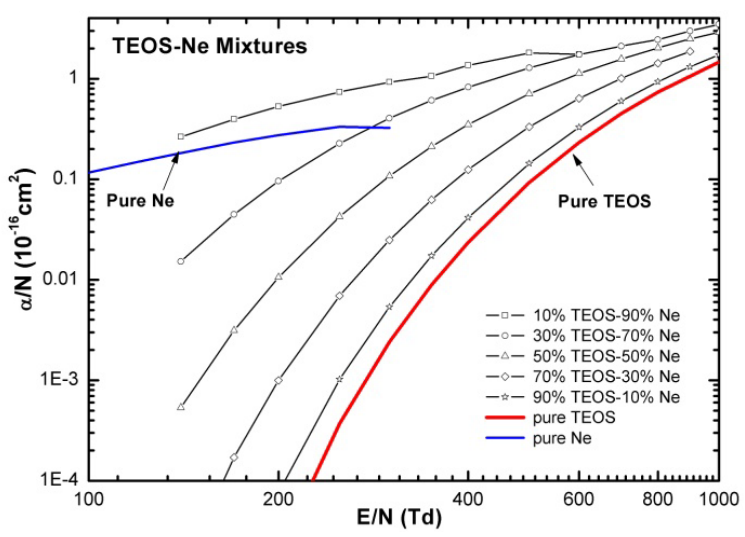

Fig. 16. The Townsend first ionization coefficients, $\alpha / \mathrm{N}$, as functions of $\mathrm{E} / \mathrm{N}$ for the TEOS-Ne mixtures with $10 \%, 30 \%, 50 \%, 70 \%$, and $90 \%$ TEOS The solid curves show present $\alpha / \mathrm{N}$ values calculated for the pure TEOS and Ne molecules. 
gas, especially on plasma assisted thin-film deposition. Especially on models for the binary mixture gases of TEOS gas with high concentration of buffer gases such as $\mathrm{Kr}$, $\mathrm{Xe}, \mathrm{He}$, and $\mathrm{Ne}$ should be experimented in gas discharge plasma conditions in order to reduce the dangers of explosion and toxicity while improving quality of the deposition of $\mathrm{SiO}_{2}$.

\section{Conclusions}

The electron transport coefficients (electron drift velocity, density-normalized longitudinal diffusion coefficient, and density-normalized effective ionization coefficient) in binary mixtures of TEOS gas with buffer gases such as $\mathrm{Kr}, \mathrm{Xe}, \mathrm{He}$, and $\mathrm{Ne}$ gases was calculated for the first time and analyzed by a two-term approximation of the Boltzmann equation in the $\mathrm{E} / \mathrm{N}$ range of $0.1-1000 \mathrm{Td}$. Models of gas discharge plasmas could be constructed using the binary mixture gases of TEOS gas with high concentration of buffer gases such as $\mathrm{Kr}, \mathrm{Xe}, \mathrm{He}$, and $\mathrm{Ne}$. These binary gas mixtures can be considered to use as the silicon sources in many industrial applications depending on mixture ratio and particular application of gas, especially on plasma assisted thin-film deposition.

\section{References}

[1] G. Tochitani, M. Shimozuma and H. Tagashira, "Deposition of Silicon Oxide Films from TEOS by Low Frequency Plasma Chemical Vapor Deposition", J. Vac. Sci. Technol. A, vol. 11, 400 (1993).

[2] K. Sano, H. Tamamaki, M. Nomura, S. Wickramanayaka, Y. Nakanishi and Y. Hatanaka, "Deposition of High Quality $\mathrm{SiO}_{2}$ Films Using Teos by ECR Plasma”, MRS Proceedings, vol. 396, 1995.

[3] J. Janča, A. Tálský and V. Zvoníček, "Kinetics of $\mathrm{O}_{2}$ + TEOS Gas-Phase Chemical Reactions in a Remote RF Plasma Reactor with Electron Spin Resonance", Plasma Chemistry and Plasma Processing, vol. 16, no. 2, 187-194, 1996.

[4] D. A. Tuan and B. H. Jeon, "Electron Collision Cross Sections for the Tetraethoxysilane Molecule and Electron Transport Coefficients in Tetraethoxysilane$\mathrm{O}_{2}$ and Tetraethoxysilane-Ar Mixtures", Journal of the Physical Society of Japan, vol. 81, no. 6, pp. 064301-1-8, Jun. 2012.

[5] D. A. Tuan and P. N. Thang, "Electron Transport Coefficients in TEOS- $\mathrm{N}_{2}$ Mixture for using in Plasma Assisted Thin-film Deposition", Journal of Science and Technology (in Thai Nguyen University), 137 (07): 121-125

[6] P. X. Hien, B. H. Jeon, and D. A. Tuan, "Electron Collision Cross Sections for the $\mathrm{BF}_{3}$ Molecule and
Electron Transport Coefficients in $\mathrm{BF}_{3}-\mathrm{Ar}$ and $\mathrm{BF}_{3}$ $\mathrm{SiH}_{4}$ Mixtures“, Journal of the Physical Society of Japan, vol. 82, no. 3, pp. 034301-1-8, Mar. 2013.

[7] P. X. Hien, D. A. Tuan, and B. H. Jeon, "Electron Collision Cross Sections for the TMS Molecule and Electron Transport Coefficients in TMS-Ar andTMSO2 Mixtures," Journal of the Korean Physical Society, vol. 61, no. 1, pp. 62-72, Jul. 2012.

[8] D. A. Tuan, "Calculations of Electron Transport Coefficients in $\mathrm{Cl}_{2}$ - $\mathrm{Ar}, \mathrm{Cl}_{2}-\mathrm{Xe}$, and $\mathrm{Cl}_{2}-\mathrm{O}_{2}$ Mixtures", Journal of the Korean Physical Society, vol. 64, no. 1, pp. 23-29, Jan. 2014.

[9] D. A. Tuan, "Analysis of Insulating Characteristics of $\mathrm{Cl}_{2}$-He Mixture Gases in Gas Discharges", J. Electr. Eng. Technol., vol. 10, no. 4, pp. 1735-1738 (Jun. 2015).

[10] M. Hayashi, "Luminous Layers in the Prebreakdown Region of Low Pressure Noble Gases," J. Phys. D, vol. 15, no. 8, pp. 1411-1418 (Aug. 1982).

[11] 11T. Hashimoto and Y. Nakamura, Papers of Gas Discharge Technical Committee, vol. ED-90-61 (Japan: IEE), 1990, as quoted in M. Suzuki, T. Taniguchi, N. Yoshimura, and H. Tagashira, "Momentum Transfer Cross Section of Xenon Deducted from Electron Drift Velocity Data," J. Phys. D, vol. 25, no. 1, pp. 50-56 (Jan. 1992).

[12] H. Tagashira, Y. Sakai and S. Sakamoto, "The Development of Electron Avalanches in Argon at High E/N Values. II. Boltzmann Equation Analysis", J. Phys. D, vol.10, 1051 (1977).

[13] D. A. Tuan, "Determination of Electron Collision Cross Sections for $\mathrm{F}_{2}, \mathrm{Cl}_{2}$ Molecules, and Electron Transport Coefficients in Mixture Gases as Pro-spective Substitutes for the $\mathrm{SF}_{6}$ Gas in Industrial Applications", PhD Dissertation, Dongguk Univ., Korea, 2012.

[14] L. G. H. Huxley and R. W. Crompton, "The Diffusion and Drift of Electrons in Gases", (New York: John Wiley \& Sons) (1974) Chaps. 6 and 13.

[15] B. H. Jeon, "Determination of Electron Collision Cross-sections for the $\mathrm{C}_{3} \mathrm{~F}_{8}$ Molecule by Using an Electron Swarm Study", J. Korean Phys. Soc. 49 (2006) 2321

[16] L. G. Christophorou and S. R. Hunter, ElectronMolecule Interations and Their Applications, vol. 2, ed L G Christophorou (Florida: Academic Press) pp. 318-412 (1984).

[17] W. L. Morgan, C. Winstead and V. McKoy, "Electron Collision Cross Sections for Tetraethoxysilane", Journal of Applied Physics, vol. 92, no. 3, pp. 16631667, Aug. 2002.

[18] J. Holtgrave, K. Riehl, D. Abner, and P. D. Haaland, "Ion Chemistry in Tetraethylorthosilicate $\left(\mathrm{C}_{2} \mathrm{H}_{5} \mathrm{O}\right)_{4} \mathrm{Si}$ ", Chemical Physics Letters, vol. 215, no. 6, pp. 548553, Dec. 1993. 


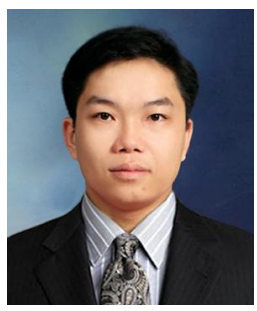

Do Anh Tuan He received the B.S and M.Sc. degrees in electrical engineering from Hanoi University of Science and Technology, Vietnam in 2004 and 2008, respectively. He received the Ph.D. degree in electrical engineering from Dongguk University, Korea in 2012. $\mathrm{He}$ is the Lecturer at the Faculty of Electronics and Electrical Engineering of Hung Yen University of Technology and Education, Vietnam from 2008. His research interests include electron swarm study, discharges and high voltage, and plasma applications. 